Fermi National Accelerator Laboratory

FERMILAB-Conf-91/196

\title{
Coil End Design for the SSC Collider Dipole Magnet
}

\author{
J. Brandt, N. Bartlett, R. Bossert, J. Carson, J. Konc, G. Lee \\ Fermi National Accelerator Laboratory \\ J. Cook \\ Argonne National Laboratory \\ S. Caspi \\ Lawrence Berkeley Laboratory \\ M. Gordon, F. Nobrega \\ SSC Laboratory
}

July 1991

* Presented at the 14th Biennial IEEE Particle Accelerator Conference, San Francisco, CA, May 6-9, 1991. 


\section{Disclaimer}

This report was prepared as an account of work sponsored by an agency of the United States Government. Neither the United States Government nor any agency thereof, nor any of their employees, makes any warranty, express or implied, or assumes any legal liability or responsibility for the accuracy, completeness, or usefullness of any information, apparatus, product, or process disclosed, or represents that its use would not infringe privately owned rights. Reference herein to any specific commercial product, process, or service by trade name, trademark, manufacturer, or otherwise, does not necessarily constitute or imply its endorsement, recommendation, or favoring by the United States Government or any agency thereof. The views and opinions of authors expressed herein do not necessarily state or reflect those of the United States Government or any agency thereof. 


\title{
Coil End Design for the SSC Collider Dipole Magnet
}

\author{
J. S. Brandt, N. W. Bartlett, R. C. Bossert, J. A. Carson, \\ J. J. Konc, G. C. Lee, Fermi National Accelerator Laboratory, \\ J. M. Cook, Argonne National Laboratory, S. Caspi, Lawrence \\ Berkeley Laboratory, M. A. Gordon, F. Nobrega, SSC Laboratory
}

\section{Abstract}

This paper describes the design of the coil end for the $50 \mathrm{~mm}$ aperture SSC collider dipole magnets built at Fermilab. The cable paths are determined from both magnetic and mechanical considerations. The end spacers are designed using the developable surface, grouped end approach, which allows the analysis of strain energy within the conductor groups. Techniques for strain energy minimisation are presented and the behavior of individual conductors within a group is analyzed. The relationship between optimixation of magnetic and mechanical variables is discussed. Requirements of manufacturing and inspection of coil end parts are outlined.

\section{Introduction}

A method termed developable surface/grouped end was chosen to be used in the design of the Fermilab-produced SSC 50mm aperture collider dipoles. Each cross section conductor block is treated as a group of conductors that maintain conductor-to-conductor contact as they wind around the coil end. The group is a mathematically determined configuration that attempts to minimize the strain energy within the group and is created by a computer program, BEND, written by Joe Cook.

\section{Group Definitions}

The group is defined within a right hand Cartesian coordinate system with the $Z$-axis located on the beam tube center line. The group originates in the $X-Y$ plane at $Z=0$ and terminates in the $Y-Z$ plane at $X=0$. Thus, only half an as-wound group is defined and is mirrored across the $Y-Z$ plane for return end groups or matched to different groups for the lead end.

The group is constrained in four ways. First, its initial direction must be parallel to the positive $Z$-axis. Second, its initial cross section in the $X-Y$ plane must match the coil cross section. Third, its final direction must be parallel to the negative $X$-axis. Fourth, all the conductors in the group must have their radially outermost edges on the outer surface of a cylindrical tube. The outer and inner radii of the tube are the outer and inner coil radii.

The group is constructed around a strip in space called the guiding strip. This is the least strain surface definable within the given constraints. The radially outer edge of the guiding strip lies on the outer surface of the tube and is called the base curve. The radially inner edge of the guiding strip is called the free edge. This edge is mathematically determined and is not constrained to a tube surface. The width of the strip is usually the difference between the outer and inner coil radii.

Fifty points are established on both the base curve and the free edge. Lines connecting like-numbered points on each edge form a unique set of surface rulings or fold lines. The guiding strip surface can be located at any conductor surface of the group, including the inside and outside group surfaces. Program BEND will refer to all three of these group surfaces. The number of conductors inside and outside the strip is a user-entered variable set related to the coil cross section and the selected guiding strip position.

Conductor-sise trapesoids are stacked against the guiding strip with one trapesoid per conductor per guiding strip point. One edge of the trapesoids adjacent to the guiding strip are coincident to the fold line at that point. The guiding strip fold lines are transferred to the inside and outside group surfaces during this process.

\section{Group Variables}

The edge of a group surface that lies in the initial $X-Y$ plane is called the initial edge, the edge that lies in the final $Y-Z$ plane is called the final edge. The angles from vertical of these two edges are called the initial edge angle and the final edge angle. The initial edge angle for any group is determined by the coil cross section geometry at the chosen guiding strip location to insure that the inside and outside group surfaces match the corresponding wedge surfaces.

The angle from vertical of a line passing through the $0,0,0$ origin and through the intersection of the initial edge and the outer tube radius is called the starting angle. This angle is also determined from the geometry of the coil cross section to ensure proper asimuthal positioning of the group.

The user is given the chance to change the distribution of twist along the strip with a variable called shift. A shift of zero is the default value and produces an even distribution of twist. Positive values of shift cause more of the twist to be distributed early in the strip, negative values distribute more twist late in the strip.

Another variable called blunt changes the radius of cur- 
vature of the free edge. A blunt of zero is the default and has no effect on the strip. Positive values of blunt pull the free edge of the strip out near its termination to relieve sharp radii at the nose of the group. Negative values pull the free edge out near its middle to improve areas between groups that may be too thin.

The magnitude of the largest $Z$-coordinate of any group surface is referred to as the $A$-length. A-lengths are defined only for points that lie on the outer surface of the tube in the final $Y-Z$ plane. Either the guiding strip A-length, the inside group A-length, or the outside group A-length may be specified by the user. This dimension is determined by both magnetic and mechanical considerations.

Magnetically, relative positions of conductor blocks in a coil end can be determined to optimize end field disturbances. A-lengths may be desired to be as small as possible to shorten the magnetic length of the end. The $0,0,0$ origin of the group does not have to be coincident to the $0,0,0$ origin of the coil end. The origin difference of a group is the distance the $X, Y, Z$ coordinate system of the group is transiated along the $Z$-axis from the $0,0,0$ origin of the coil end.

Certain mechanical considerations may require variation of the desired magnetic configuration. Program BEND distributes the group's twist in an attempt to minimize the strain energy within the group. An A-length may be too short to smoothly distribute the group's twist. The guiding strip may buckle or fold back on itself due to crossing of fold lines. The user should carefully analyze groups that are hard to optimize.

\section{Conductor Variables}

Conductor width will be the same as the specified guiding strip width. Conductor mid-thickness should be the same as the average mid-thickness of the conductors within the group. If a less tightly compressed configuration is desired, cable-thickening ratios greater than one can be specified that increase the cable mid-thickness at the middle of the group, at the termination of the group, or both. The mid-thickness at the origin of the group will remain unchanged with the specified cable-thickening ration smoothly applied from there.

The use of the group's average conductor keystone angle will result in exact matching to the appropriate wedge surfaces. Just as the mid-thickness of the conductor can be altered, so can the keystone angle. Keystone-widening ratios less than one will decrease the conductor keystone angle according to the same constraints as outlined for mid-thickness.

Groups defined for the SSC $40 \mathrm{~mm}$ magnets used the above conductor modification ratios set to one, specifying the same individual conductor geometry in the end group as in the coil cross section. Study of these ends showed that the conductor keystone angle appeared to be less in the ends than in the straight section. It also appeared that the group was more tightly compressed in the middle than at the nose. Based on these observations, keystone-widening ratios of 0.600 and 0.700 and cable-thickening ratios of
1.027 and 1.000 were used in the SSC $50 \mathrm{~mm}$ design.

Analysis of the first short SSC $50 \mathrm{~mm}$ ends shows that slightly lower keystone-widening ratios may be an improvement. Inability to close the coil ends to their designed positions without damage to individual conductor insulation has indicated that changes in the cable-thickening ratios are also in order. The specification of larger cablethickening ratios will result in a coil end that closes to its designed length at the expense of less conductor compaction in the end groups.

\section{Group Optimization}

Use of the two variables, shift and blunt, will affect the geometry and the strain energy of the guiding strip, negatively in normal groups. However, optimization of these variables can have a pronounced positive effect on the strain energy within the group, particularly on conductors which are farther away from the guiding strip. Shift variation should always be attempted with blunt variation saved for problem configurations.

During this interactive process, the user will attempt to minimize the $\Delta L / L$ within the group. If the radius of curvature of the three group surfaces is considered to be too small, some experimentation with blunt or a change in guiding strip position may be required. Different guiding strip positions can also have a dramatic affect on the $\Delta L / L$ within the group. By empirical analysis, a maximum $\Delta L / L$ of 0.3 and a minimum radius of curvature $2.5 \mathrm{~mm}$ are considered acceptable limits.

Upon user acceptance of an optimized configuration, BEND offers a chance to save group defining files. These six files will for the most part contain $X, Y, Z$ coordinates of points and will be named by a user-entered file-name and given a three digit file-type. The .COR file contains four corner points for each conductor trapezoid at each fold line. The .CEN file contains the centroid for all conductor traperoids. The .FRA file contains the Frenet frame for each trapezoid. The .INS file contains points defining the inside surface of the group where the fold lines intersect the tube radii. The .OUS file contains points defining the outside surface of the group where the fold lines intersect the tube radii and also contains points defining shelf undercut. The .SHF file contains points defining the group's shelf and shelf extension.

\section{Part Definitions}

The last three BEND output files mentioned above define the boundary surfaces of a group. These files contain fifty $X, Y, Z$ coordinate points for each edge of each surface specified in the file. Coil end parts containing these boundary surfaces are manufactured to constrain the group of conductors to its designed configuration.

Parts used on the return end of a coil are defined by group surfaces which are mirrored across the $Y-Z$ plane. Lead end parts are not symmetrical in this way and must contain group boundary surfaces with both positive and negative $X$-coordinates. Normally, a return end group is constructed first and and used to define half of the corre- 
sponding lead end group. New lead end groups are then constructed to match the return end group in the final $Y$ $Z$ plane. Negative signs must be added to the appropriate $X$-coordinates.

A ten-conductor return end group will typically require a nine-conductor lead end group to match on its inside surface at the final plane and a one-conductor lead end crossover group to match on its outside surface in the final $Y-Z$ plane. The shelf under the lead end groups would contain the rearmost surface of the nine-conductor group and the leading surface of the one-conductor group.

The one-conductor group is used to switch the winding from one conductor block to the next. To continue the above example, the filler is defined by the .OUS surface of the nine-conductor lead end group and the .INS surface of the one-conductor group. The one-conductor group should contain approximately the same twist distribution as the nine-conductor group to allow a good fit on the composite shelf used under them.

\section{Part Descriptions}

The first group in a coil end is wound around a part called a key. A key is defined by a group.INS file and may require a shelf to be attached to it.

A shelf is defined for all groups whether it is needed or not. If the final edge angles of the group are large enough, the radially inner edges of the conductors will have lifted from the inner tube radius to form a space of varying thickness which is filled by the shelf. The rearmost surface of the shelf is defined by its intersection with the .INS surface while its leading surface is defined by the shelf's intersection with the .OUS surface.

The leading surface also has an arbitrary positive $Z$ axis extension applied to it. This extension is necessary to prevent uncompressed conductors from overhanging the shelf during the winding process. Overhanging conductors can be damaged in curing while being pinched between end parts. The shelf extension requires that the part in front of it be undercut to allow the part to slide over the extension during the curing process.

A part called a spacer is installed around a previouslywound group and forms the winding surface for the next group. A spacer fills the space between each group and is defined by the .OUS surface of the group behind it and the .INS surface of the group in front.

The spacers for the SSC $50 \mathrm{~mm}$ dipoles are very stiff by design because of the desire to have a dowel pin through the part used for machining, inspection, and assembly positioning. This stiffness causes problems in the winding process when the spacers are installed around uncompressed groups of conductors. The danger of damaged conductor insulation and the lift of the shelves from the mandrel prevent the spacers from being installed as tightly around the group as they could be. This results in the next-wound group being positioned farther from its desired cured position. All $50 \mathrm{~mm}$ spacers used at Fermilab are being slotted by thin saw blade at two or three places on their outer surface to make them more flexible and easy to position during the winding process.

A part called a saddle is installed around the last-wound group and provides a surface through which hydraulic pressure to close the end is applied while curing.

The final end part type is called a filler. A filler is used only on the lead end of the coil to support the oneconductor crossover group.

\section{Part Manufacture}

Molded end parts are being tested but parts used in the first $50 \mathrm{~mm}$ coils will be machined from a G-10 tube. Acceptable end parts must contain surfaces based on curves through the part data points and containing the unique set of fold lines generated from them.

The variation of cutting tool angle sequired to produce this cut is beyond the limits of some five-axis machines. The integrity of all surfaces must be maintained during the machining of adjacent surfaces. These constraints have forced the machining of most parts to be done in multiple passes.

Surfaces that feather to zero thickness are encountered on all shelves, fillers, and lead end spacers. Most manufacturers can feather to about $0.13 \mathrm{~mm}$ before the part material begins to break up due to machining forces.

Shelf extensions and undercuts originate at the curve that is the intersection of the top shelf surface and the outside group surface. Points on this curve are translated in the positive $Z$ direction to a place where the perpendicular length of the extension was approximately $3.18 \mathrm{~mm}$ all around the shelf. Undercut points were arbitrarily given an additional $0.38 \mathrm{~mm} Z$ direction translation. The fold lines of an extension or an undercut surface consist of rulings parallel to the $Z$-axis and can be cut with the side of a cylindrical cutter. The corresponding undercut is toleranced to allow for increased $Z$-axis depth to avoid interference between the tool fillet produced by this cut and the leading surface of the shelf extension

\section{Part Inspection}

A complete set of inspection fixturing has been designed at Fermilab to provide accurate and repeatable $50 \mathrm{~mm}$ coil end part inspection. A part is installed in a measuring apparatus that uses a small spherical probe to contact the part at known nominal point positions produced by a computer program written by John Kone and Greg Lee. The movement of the probe as it checks each part surface is controlled by computer and inspection data is automatically produced.

\section{Conclusion}

The developable surface/grouped end approach specified for the SSC $50 \mathrm{~mm}$ aperture collider dipole magnets has allowed the design of a coil end that is not only the least strain configuration but also provides good placement and containment of each conductor in the cross section. Magnetic and mechanical considerations have be implemented and analyzed and part inspection requirements are fully defined. An expanded version of this paper with illustrations and references is available as Fermilab-TM-1735. 